pag

Business School

WORKING PAPER SERIES

\begin{tabular}{c|l} 
Working Paper & $\begin{array}{l}\text { Pareto optima and equilibria when } \\
\text { preferences are incompletely known }\end{array}$ \\
$2014-060$ & $\begin{array}{l}\text { G. Carlier } \\
\text { R.-A. Dana }\end{array}$
\end{tabular}

http://www.ipag.fr/fr/accueil/la-recherche/publications-WP.html

IPAG Business School

184, Boulevard Saint-Germain

75006 Paris

France

IPAG working papers are circulated for discussion and comments only. They have not been peer-reviewed and may not be reproduced without permission of the authors. 


\title{
Pareto optima and equilibria when preferences are incompletely known
}

\author{
G. Carlier ${ }^{*}$ R.-A. Dana ${ }^{\dagger}$
}

March 20, 2013

\begin{abstract}
An exchange economy in which agents have convex incomplete preferences defined by families of concave utility functions is considered. Sufficient conditions for the set of efficient allocations and equilibria to coincide with the set of efficient allocations and equilibria that result when each agent has a utility in her family are provided. Welfare theorems in an incomplete preferences framework therefore hold under these conditions and efficient allocations and equilibria are characterized by first order conditions.
\end{abstract}

Keywords: incomplete preferences, efficient allocations and equilibria.

\section{Introduction}

Since the work of von Neumann and Morgenstern [32], a number of authors have raised objections about the use of the completeness axiom in utility theory (for early work, see for example Luce and Raiffa [20], Aumann [3], Shapley [31]). These objections have on one hand, lead to the development of axiomatic theories and multi-utility representations of incomplete preferences (see for example Aumann [3], Dubra et al. [13], Maccheroni [21], Ok [24]). Incomplete preferences have, on the other hand, been used in a wide variety of fields, for example, risk theory (Rothschild and Stiglitz [27]), finance

\footnotetext{
${ }^{*}$ CEREMADE, UMR CNRS 7534, Université Paris IX Dauphine, Pl. de Lattre de Tassigny, 75775 Paris Cedex 16, FRANCE carlier@ceremade.dauphine.fr

${ }^{\dagger}$ CEREMADE, UMR CNRS 7534, Université Paris IX Dauphine, Pl. de Lattre de Tassigny, 75775 Paris Cedex 16, FRANCE dana@ceremade.dauphine.fr , G. Carlier and R.-A. Dana acknowledge the support of the Fondation du Risque, chaire Dauphine-ENSAE-Groupama, "Les particuliers face au risque" and of the ANR project Risque.
} 
(for example Dybvig [14], Jouini and Kallal [19], Easley and O'Hara [15]), noncooperative game theory (Bade [4], Evren [16]), asymmetric information (no-trade results of Samet [30] and Man-Chung Ng [22]) and equilibrium theory (Mas-Colell [23], Gale and Mas-Colell [18]). Recently there has been a renewed interest for incomplete preferences with the work of Bewley [6] and Rigotti and Shannon [28] as a way to model choices in the presence of ambiguity and to explain non participation in markets for which there is ambiguity and more particularly in financial markets (see Dana and Riedel [12] and Easley and O'Hara [15]).

This paper considers an exchange economy populated by a finite number $I$ of agents whose preferences are incompletely known. Their utilities may for example depend on unknown parameters or more generally belong to given families $\mathcal{U}_{i}, i \in I$ of utility functions. For each $\mathcal{U}_{i}$, the corresponding strict preference is defined by the unanimity rule: $X \in E$ is strictly preferred to $Y \in E$ (which will be denoted $X \succ_{\mathcal{U}_{i}} Y$ ) if $u(X)>u(Y)$ for every $u_{i} \in \mathcal{U}_{i}$. It is worth pointing out that there are two interpretations to this setting. In the first one, agents may have complete preferences but the analyst does not know every ranking ${ }^{1}$. An alternative interpretation is in assuming that agents do not rank some alternatives and that this is known by the analyst, it is this interpretation that relates the present paper to the literature on incomplete preferences.

The purpose of the paper is to identify conditions under which the following two properties are satisfied:

- efficient allocations for the incomplete preferences coincide with the set of efficient allocations that result for some choice of utilities in the sets of each agent,

- equilibria for the incomplete preferences coincide with the set of equilibria that result for some choice of utilities in the sets of each agent.

A positive answer to the previous questions has numerous implications. It, in particular, implies that the welfare theorems hold true in an incomplete preferences framework and that efficient allocations may be characterized by first order conditions (namely the intersection of normalized supergradients is non empty for some choice of utilities in the sets of each agent).

The paper considers concave multi-utility representations. This covers the case of families of linear preferences often used in asymmetric information models, of second order stochastic dominance (Rothschild and Stiglitz [27]), of Bewley's [6] and Rigotti and Shannon [28] ambiguity models with

\footnotetext{
${ }^{1}$ This is actually this interpretation the term incompletely known in the title refers to.
} 
incomplete preferences. It is based on a definition of efficiency corresponding to the strict preferences defined above and provides sufficient conditions for obtaining a positive answer to both of the above questions. In particular compactness conditions on the set of utility functions and on the set of their super differentials at any point are required. We also show that the above questions have a positive answer in the case of second order stochastic dominance for univariate risks although our technical assumptions are not satisfied in this case.

The next example shows that one must consider convex families of utilities $\mathcal{U}_{i}$ whatever strict order or definition of efficiency and equilibria is chosen ${ }^{2}$. Indeed, consider the case of two agents sharing risk $X$, the first agent has the CARA utility $X_{1} \mapsto-\mathbb{E}\left(e^{-X_{1}}\right)$ and the second one has incomplete preferences given by the family $\mathcal{U}_{2}$ of CARA utilities $X_{2} \mapsto-\mathbb{E}\left(e^{-\theta X_{2}}\right)$ where $\theta \in[1 / 2,2]$. Note that $\mathcal{U}_{2}$ is not a convex family. Efficient risk sharing pairs between the first agent and any agent with utility in $\mathcal{U}_{2}$ are pairs of affine functions of $X$, with slopes $\left(\frac{\theta}{1+\theta}, \frac{1}{1+\theta}\right), \theta \in[1 / 2,2]$. Consider the following efficient sharing problem between agent one and an agent with utility $-\frac{1}{5} \mathbb{E}\left(e^{-2 X_{2}}\right)-\frac{4}{5} \mathbb{E}\left(e^{-\frac{X_{2}}{2}}\right)$, obtained by mixing two utilities in $\mathcal{U}_{2}$

$$
\max _{X_{1}+X_{2}=X}-\mathbb{E}\left(e^{-X_{1}}\right)-\frac{1}{5} \mathbb{E}\left(e^{-2 X_{2}}\right)-\frac{4}{5} \mathbb{E}\left(e^{-\frac{1}{2} X_{2}}\right) .
$$

The solution of (1.1) is undominated in the incomplete setting (see definition 3.4 below $)$ : there does not exist $\left(X_{1}^{\prime}, X_{2}^{\prime}\right)$ such that $-\mathbb{E}\left(e^{-X_{1}^{\prime}}\right)>-\mathbb{E}\left(e^{-X_{1}}\right)$ and $-\mathbb{E}\left(e^{-\theta X_{2}^{\prime}}\right)>-\mathbb{E}\left(e^{-\theta X_{2}}\right)$ for any $\theta \in[1 / 2,2]$. However $X_{1}$ which is implicitely defined by the equation $e^{-X_{1}}+\frac{2}{5} e^{-2\left(X-X_{1}\right)}+\frac{2}{5} e^{-\frac{1}{2}\left(X-X_{1}\right)}=0$ is not an affine function of $X$. Therefore it cannot be obtained as an efficient allocation that result when each agent has a utility in her family.

The method used is to first establish a no trade principle for families of linear utilities parametrized by a family of convex compact subsets of a given hyperplane by using an extension of Samet's results [30]. From the no-trade principle, any feasible allocation is efficient if and only if the compact sets have non-empty intersection. A characterization of efficient allocations for families of concave utilities is, then obtained by local linearization of utilities. Thus the paper connects results that have been proven in the no trade literature (Samet [30], Man-Chung Ng [22]) mainly for linear utilities and infinite dimensional generalizations of results proven in the general equilibrium literature for concave utilities (Rigotti and Shannon's [28] ambiguity model with incomplete preferences).

\footnotetext{
${ }^{2}$ In a linear framework, the crucial role played by the convexity of the multi-utility sets was already emphasized in Carroll [9].
} 
The paper is organized as follows. The framework and general assumptions and examples are presented in section 2. Section 3 provides an abstract no-trade principle which nests most of the ex-ante no-trade theorems existing in the literature. Efficiency is then characterized and efficient allocations for the incomplete preferences are shown to coincide with the set of efficient allocations that result when each agent has a utility in her family of concave utility functions. A characterization by first order condition is provided. Section 4 is devoted to equilibria and welfare theorems. Section 5 discusses some concepts used in the paper and dicusses relations to the literature.

\section{Framework, assumptions and examples}

\subsection{Incomplete preferences framework}

Let $E$ and $F$ be two real vector spaces, and $(P, X) \in F \times E \mapsto P \cdot X \in \mathbb{R}$ be some separating duality mapping which means that this map is bilinear and

- if $X \in E$ is such that $P \cdot X=0$ for all $P \in F$ then $X=0$,

- if $P \in F$ is such that $P \cdot X=0$ for all $X \in E$ then $P=0$.

We endow $F$ (respectively $E$ ) with the locally convex Hausdorff topology $\sigma(F, E)$ (resp. $\sigma(E, F)$ ) which is the coarsest topology on $F$ (resp. on $E$ ) for which $P \in F \mapsto P \cdot X$ is continuous for every $X \in E$ (resp. $X \in E \mapsto P \cdot X$ is continuous for every $P \in F$ ). With this choice of topologies, the topological dual of $F$ may be identified to $E$ and vice versa (see for instance Aliprantis and Border [1], Theorem 5.83). We shall therefore in the sequel interpret $E$ as the space of goods and $F$ as the space of prices.

Two polar special cases will mainly be considered. In the first, $E$ is a Banach space, $F=E^{\prime}$ and $\sigma(F, E)$ is the weak star topology on $F=E^{\prime}$ (examples are $(E, F)=\left(L^{1}, L^{\infty}\right)$ or $E$ is a space of continuous functions and $F$ a space of Radon measures). In the polar case, $F$ is a Banach space, $E=F^{\prime}$ and $\sigma(F, E)$ is the weak topology on $F$ (a typical example being $E=L^{\infty}$ and $\left.F=L^{1}\right)$. An example covered by our framework but not by the previous two cases is $E=B(\Omega, \mathcal{F})$ the space of real-valued bounded measurable functions on $(\Omega, \mathcal{F})$, a measurable space, $E^{\prime}=\mathrm{ba}(\Omega, \mathcal{F})$ the space of finitely additive measures on $(\Omega, \mathcal{F})$ and $F=\mathrm{ca}(\Omega, \mathcal{F})$ the subspace of countably additive measures on $(\Omega, \mathcal{F})$.

An exchange economy with consumption space $E$ populated by a finite set $I$ of agents is considered. Agent $i \in I$ has an incomplete strict preference over $E$, defined by a family $\mathcal{U}_{i}: E \rightarrow \mathbb{R}$ of utility functions as follows: $X_{i} \in E$ 
is strictly preferred to $Y_{i} \in E$ by agent $i$, which will be denoted $X_{i} \succ_{\mathcal{U}_{i}} Y_{i}$, if $u_{i}\left(X_{i}\right)>u_{i}\left(Y_{i}\right)$ for every $u_{i} \in \mathcal{U}_{i}$.

For $X \in E$ (aggregate endowment), the set of allocations of $X$ are defined as:

$$
\mathcal{A}(X):=\left\{\left(X_{i}\right)_{i \in I} \in E^{I}: \sum_{i \in I} X_{i}=X\right\} .
$$

An allocation $\left(X_{i}\right)_{i \in I} \in \mathcal{A}(X)$ is efficient if there is no other allocation $\left(Y_{i}\right)_{i \in I} \in \mathcal{A}(X)$ fulfilling $Y_{i} \succ_{\mathcal{U}_{i}} X_{i}$ for every $i \in I .{ }^{3}$

For further use, we recall that for $u: E \rightarrow \mathbb{R}$ concave, the superdifferential of $u$ at $X \in E$, denoted $\partial u(X)$, is defined by

$$
\partial u(X):=\left\{P \in E^{\prime}=F: u(Y)-u(X) \leq P \cdot(Y-X) \text {, for all } Y \in E\right\}
$$

and that $u$ is superdifferentiable at $X$ if $\partial u(X)$ is nonempty. We shall also denote

$$
\partial \mathcal{U}_{i}(X):=\bigcup_{u_{i} \in \mathcal{U}_{i}} \partial u_{i}(X)
$$

Note that as

$$
\lambda \partial u_{i}(X)+(1-\lambda) \partial v_{i}(X) \subset \partial\left(\lambda u_{i}+(1-\lambda) v_{i}\right)(X), 0 \leq \lambda \leq 1, X \in E
$$

$\partial \mathcal{U}_{i}(X)$ is convex whenever $\mathcal{U}_{i}$ is convex. In the sequel, unless otherwise stated, the following assumptions are assumed to hold:

- (H1) For every $i \in I$, utilities in $\mathcal{U}_{i}$ are everywhere finite, concave superdifferentiable functions defined on $E$. Furthermore, for every $i$, every $u_{i} \in \mathcal{U}_{i}$ and every $X \in E, \partial u_{i}(X)$ is $\sigma(F, E)$ compact.

- (H2) For every $i \in I$, the set of utilities $\mathcal{U}_{i}$ is convex and there is a topology on $\mathcal{U}_{i}$ which makes it compact and such that the evaluation map $u_{i} \in \mathcal{U}_{i} \mapsto u_{i}(X)$ is continuous for every $X \in E$.

- (H3) There exists $\Phi \in E$ such that, for every $i \in I$, every $X_{i} \in E$, every $u_{i} \in \mathcal{U}_{i}$ and every $P \in \partial u_{i}\left(X_{i}\right)$, one has $\Phi \cdot P>0$ and the set of normalized marginal utilities

$$
V_{i}\left(X_{i}\right):=\left\{\frac{P}{\Phi \cdot P}: P \in \partial \mathcal{U}_{i}\left(X_{i}\right)\right\}
$$

is $\sigma(F, E)$ compact for every $i$ and every $X_{i} \in E$.

\footnotetext{
${ }^{3}$ Other concepts of efficiency will be discussed in section 5 .
} 
Classes of examples where the previous assumptions are satisfied are provided in the next subsection. Let us make some general comments. Regarding assumption $(\mathbf{H 1})$, it always holds true if $E$ is a normed space, $F=E^{\prime}$ and the elements of $\mathcal{U}_{i}$ are Gâteaux-differentiable. More generally, $(\mathbf{H 1})$ is satisfied if $E$ is a Banach space with dual $F$ and the functions in $\mathcal{U}_{i}$ are concave, u.s.c (for the strong topology) and finite everywhere. Indeed, they are then everywhere continuous and superdifferentiable (see Chapter 1 in [17]). Moreover, their superdifferential being weakly star closed and bounded in $E^{\prime}$ is $\sigma(F, E)$ compact from Banach-Alaoglu's Theorem. In (H2), the requirement that $\mathcal{U}_{i}$ is convex has already been discussed in the introduction. Loosely put, the second part of (H2) says that the set of utilities admits a compact parametrization. To illustrate $(\mathbf{H 3})$, let $(\Omega, \mathcal{F}, \mathbb{P})$ be given and assume that there is one good in each state of the world. Let $E=L^{\infty}$ be the set of contingent claims, $F=L^{1}$ and $\Phi=1$. Then

$$
V_{i}\left(X_{i}\right)=\left\{\frac{P}{\mathbb{E}(P)}, P \in \partial \mathcal{U}_{i}\left(X_{i}\right)\right\}
$$

is the familiar set of normalized supergradients (or marginal utilities) of the $u_{i}$ 's at $X_{i}$. If in addition, utilities in $\mathcal{U}_{i}$ are nondecreasing, elements of $V_{i}\left(X_{i}\right)$ are probability densities which may therefore be interpreted as subjective beliefs as in [29].

\section{$2.2 \quad$ Examples}

In this subsection, we show that the assumptions above are satisfied in several quite large classes of examples. For the sake of notational simplicity, we will drop the subscript $i$ everywhere in these examples.

\section{The case of finite dimensions}

Let $E=F=\mathbb{R}^{d}$ and $\mathcal{U}$ be a convex set of concave functions: $\mathbb{R}^{d} \rightarrow \mathbb{R}$. We claim that (H1) is trivially satisfied. Indeed the elements of $\mathcal{U}$ are everywhere continuous and thus superdifferentiable everywhere with a superdifferential which is closed and bounded, hence compact at every point.

Let us further assume that for every $R>0$

$$
M_{R}:=\sup \{|u(x)|,|x| \leq R, u \in \mathcal{U}\}<+\infty
$$

and that $\mathcal{U}$ is closed for the topology of $C\left(\mathbb{R}^{d}\right)$ (that is that of uniform convergence on compact subsets). This implies that (H2) is fulfilled and 
that $\partial \mathcal{U}(x)$ is compact. Indeed for every $R>0$, every $u \in \mathcal{U}$, every $(x, h) \in$ $\mathbb{R}^{d} \times \mathbb{R}^{d}$ such that $|x| \leq R,|h| \leq R$ and every $p \in \partial u(x)$, we have

$$
p \cdot h \leq u(x)-u(x-h) \leq 2 M_{2 R}
$$

hence $|p| \leq K_{R}=2 M_{2 R} / R$ which proves that $\partial \mathcal{U}(x)$ is included in the ball of radius $K_{R}$ and also that $u$ is $K_{R}$ Lipschitz on the ball of radius $R$. From Ascoli's theorem, as $\mathcal{U}$ is closed, $\mathcal{U}$ is compact in $C\left(\mathbb{R}^{d}\right)$ and (H2) is fulfilled. Let us next show that $\partial \mathcal{U}(x)$ is closed. Let $p_{n} \in \partial u_{n}(x)$ converges to some $p$. Passing to a subsequence if necessary, we may assume that $u_{n}$ converges uniformly on every compact to some $u \in \mathcal{U}$. Since for every $y$, we have $u_{n}(y)-u_{n}(x) \leq p_{n}(x-y)$, letting $n$ tend to $+\infty$ gives that $p \in \partial u(x) \subset$ $\partial \mathcal{U}(x)$.

Finally, let us assume that there is a common increasing direction, i.e. some $e \in \mathbb{R}^{d}$ such that $e \cdot p>0$ for every $(x, u) \in \mathbb{R}^{d} \times \mathcal{U}$ and every $p \in \partial u(x)$ and let $V(x)$ be defined as above by

$$
V(x):=\{p /(e \cdot p), p \in \partial \mathcal{U}(x)\}
$$

As $V(x)$ is the image of the compact set $\partial \mathcal{U}(x)$ by $p \mapsto p /(e \cdot p)$ which is continuous on $\partial \mathcal{U}(x)$, it is compact and (H3) holds true.

This example shows that the assumptions made in the paper are rather harmless in finite dimensions.

\section{Linear utilities and expectations with respect to a family of priors}

Let $\Phi \in E$ and $K$ be a convex $\sigma(F, E)$ compact subset of $F$ such that $K \subset\{P \in F: P \cdot \Phi=1\}$. Consider the family of linear utilities:

$$
u_{a}(X)=a \cdot X, a \in K, X \in E \text { and } \mathcal{U}:=\left\{u_{a}, a \in K\right\}
$$

Identifying $\mathcal{U}$ to $K$ (endowed with $\sigma(F, E)$ ), as $\partial \mathcal{U}(X)=K \subset\{P \in F$ : $P \cdot \Phi=1\} ; V(X)=K$ and it is immediate to check that (H1), (H2) and (H3) are fulfilled.

Let us now consider an example. Let $A$ be a compact subset of $\mathbb{R}, E=$ $C(A, \mathbb{R})$ and $F=E^{\prime}=\mathcal{M}(A)$. One may interpret $A$ as the set of states of the world and $E$ as the set of contingent claims. Let $K$ be a convex and weakly-star closed subset of the set of probability measures on $A$. Let

$$
u_{\mu}(X):=\int_{A} X(x) d \mu(x), X \in E, \mu \in K
$$

be the expectation of $X$ with respect to the family $K$ of probabilities and let

$$
\mathcal{U}:=\left\{u_{\mu}, \mu \in K\right\}
$$


(H1) is trivially fulfilled. Identifying $\mathcal{U}$ to $K$ endowed with the weak-star topology, (H2) is fulfilled. Finally, choosing $\Phi=1$, as $\partial u_{\mu}(X)=\{\mu\}$, $V(X)=K$ for all $X$ and (H3) is in turn fulfilled.

A nonlinear variant of this model satisfying our assumptions as well, is obtained by considering families of utilities of the form

$$
v_{\mu}(X):=\int_{A} V(X(x)) d \mu(x), X \in E, \mu \in K
$$

where $V$ is a given concave differentiable utility index and $\mu$ ranges over a convex and weakly-star closed set of probability measures $K$. As $v_{\mu}$ is differentiable $\left(\partial v_{\mu}(X)=\left\{V^{\prime}(X) \mu\right\}\right),(\mathbf{H} 1)$ is trivially fulfilled. Identifying $\mathcal{U}$ to $K$ endowed with the weak-star topology, (H2) is fulfilled. Let $\Phi=1$, then $V(X)=\left\{\frac{V^{\prime}(X) \mu}{\mathbb{E}_{\mu}\left(V^{\prime}(X)\right)}, \mu \in K\right\}$ is $\sigma(F, E)$ compact and (H3) is fulfilled. This incomplete preference may be viewed as an infinite dimensional extension of Bewley's [6] and Rigotti and Shannon's [28] ambiguity incomplete preference.

Other families of linear or non linear utilities may be interpreted as expectations with respect to a family of priors. For example, one may consider $E=B(\Omega, \mathcal{F})$ and $F=$ ba $(\Omega, \mathcal{F})$ and $K$ a weak-star compact subset of probability measures. Fixing a probability $P$, one may also consider pairs such as $(E, F)=\left(L^{p}, L^{p^{\prime}}\right)($ with $p \in(1, \infty)),(E, F)=\left(L^{1}, L^{\infty}\right),(E, F)=\left(L^{\infty}, L^{1}\right)$ and $K$ a $\sigma(F, E)$ compact subset of probability densities. Nonlinear variants of these models also provide infinite dimensional extensions of Bewley's [6] and Rigotti and Shannon [28]'s ambiguity models (see Dana and Riedel [12] for a dynamic example).

\section{Incomplete preferences on random vectors}

Let $(\Omega, \mathcal{F}, \mathbb{P})$ be a probability space. We now consider strict orders on random vectors defined by families of expected utilities. More precisely, let $p \in$ $(1, \infty)$ with conjugate exponent $p^{\prime}=p /(p-1)$ and $E=L^{p}\left((\Omega, \mathcal{F}, \mathbb{P}), \mathbb{R}^{d}\right)$ and $E^{\prime}=F=L^{p^{\prime}}\left((\Omega, \mathcal{F}, \mathbb{P}), \mathbb{R}^{d}\right)$ with the usual duality map between these spaces. As the class of concave functions does not satisfy our assumptions, let us consider a class $\mathcal{V}$ of concave and $C^{1}$ functions $\mathbb{R}^{d} \rightarrow \mathbb{R}$ such that:

1. $\mathcal{V}$ is convex and closed for the topology of $C\left(\mathbb{R}^{d}\right)$ (i.e. uniform convergence on compact sets),

2. The utilities and their gradients fulfill a uniform growth conditions: there is a constant $C$ such that

$$
|v(x)| \leq C\left(|x|^{p}+1\right),|\nabla v(x)| \leq C\left(|x|^{p-1}+1\right), \forall(x, v) \in \mathbb{R}^{d} \times \mathcal{V},
$$


3. The utilities and their gradients are equicontinuous on compact sets: for every compact subset of $\mathbb{R}^{d}, K$, one has

$$
\lim _{\varepsilon \rightarrow 0^{+}} \sup _{(v, x, y) \in \mathcal{V} \times K^{2},|x-y| \leq \varepsilon}\{|v(x)-v(y)|+|\nabla v(x)-\nabla v(y)|\}=0 .
$$

4. there exists a unit vector $e \in \mathbb{R}^{d}$ such that $e \cdot \nabla v(x)>0, \forall(v, x) \in$ $\mathcal{V} \times \mathbb{R}^{d}$.

Note that from Ascoli's theorem, assumptions 1 and 3 guarantee that $\mathcal{V}$ is compact for the topology of $C^{1}\left(\mathbb{R}^{d}\right)$.

Let $\mathcal{U}$ be the set of expected utilities generated by $\mathcal{V}$ i.e.

$$
\mathcal{U}:=\left\{u_{v}:=\mathbb{E}(v(.)), v \in \mathcal{V}\right\}
$$

Assumption 2 insures that $u_{v}$ and $\nabla u_{v}$ are well defined. Let $\mathcal{U}$ be endowed with the topology induced by the topology of $C\left(\mathbb{R}^{d}\right)$ on $\mathcal{V}$. From assumption 2 and Lebesgue dominated convergence theorem, the evaluation maps $v \mapsto$ $\mathbb{E}(v(X))$ are continuous and the elements of $\mathcal{U}$ are continuous for the strong topology of $E$. Hence (H1) and (H2) are fulfilled. Let us check that (H3) is satisfied when one takes $P \cdot \Phi:=\mathbb{E}(e \cdot P)$. For $X \in E$, let

$$
V(X):=\left\{\frac{\nabla v(X)}{\mathbb{E}(e \cdot \nabla v(X))}, v \in \mathcal{V}\right\}
$$

Note that $V(X)$ is convex. We claim that $V(X)$ is strongly compact in $F$. Indeed, let

$$
Y_{n}=\lambda_{n} \nabla v_{n}(X), \lambda_{n}:=\frac{1}{\mathbb{E}\left(e \cdot \nabla v_{n}(X)\right)}
$$

be some sequence in $V(X)$. From assumption 3 and Ascoli's theorem, a subsequence $\left(v_{n}, \nabla v_{n}\right)$ converges in $C\left(\mathbb{R}^{d}\right)$ to some $(v, \nabla v)$ with $v \in \mathcal{V}$. Using again assumption 3 and Lebesgue's dominated theorem, we deduce that $\nabla v_{n}(X)-\nabla v(X)$ converges to 0 strongly in $F$. This implies that $\lambda_{n}$ also converges and that $Y_{n}$ converges strongly in $F$ to

$$
\frac{\nabla v(X)}{\mathbb{E}(e \cdot \nabla v(X))} .
$$

Since $V(X)$ is convex, we thus have (H3).

Families of state dependent, additively separable utilities on random vectors

$$
u_{v}(X):=\int_{\Omega} v(\omega, X(\omega)) d \mathbb{P}(\omega) .
$$


may as well be considered (H1), (H2) and (H3) are fulfilled under similar assumptions as above, uniform in $\omega$.

In the previous examples, except in the finite dimensional case, utilities were assumed differentiable. Let us now consider an example of families of superdifferentiable utilities.

\section{Families of Rank-linear Utilities}

Let $(\Omega, \mathcal{F}, \mathbb{P})$ be a non-atomic probability space, $E=L^{\infty}((\Omega, \mathcal{F}, \mathbb{P}), \mathbb{R})$ and $F=L^{1}((\Omega, \mathcal{F}, \mathbb{P}), \mathbb{R})$. Rank-linear-utilities both generalize expected utilities and Choquet's integrals with respect to a distortion and are of the form

$$
X \in E \mapsto u_{l}(X):=\int_{0}^{1} l\left(t, F_{X}^{-1}(t)\right) d t
$$

where $F_{X}^{-1}=\inf \left\{z \in \mathbb{R}: F_{X}(z)>t\right\}$ denotes the quantile of $X$ and $l$ is a smooth function, concave nondecreasing in its second argument and submodular (i.e. $\partial_{t x}^{2} l \geq 0$ ). The expected utility is obtained for $l(t, x)=v(x)$ and $v: \mathbb{R} \rightarrow \mathbb{R}$ concave nondecreasing, the Choquet expectation with respect to a convex $C^{1}$ distortion $f$ for $l(t, x)=f^{\prime}(1-t) x$ and the risk averse RDU for $l(t, x)=f^{\prime}(1-t) v(x)$ with $f$ convex and $v$ concave nondecreasing.

Let us consider the family of utilities

$$
\mathcal{U}:=\left\{u_{l}, l \in \mathcal{L}\right\}
$$

where $\mathcal{L}$ is a convex and compact (for the $C([0,1] \times \mathbb{R})$ topology) set of $C^{1}$ concave and nondecreasing in their second argument functions fulfilling $\partial_{t x}^{2} l>0$. We identify $\mathcal{U}$ with $\mathcal{L}$. From a supermodular version of HardyLittlewood's theorem, one has for every $l \in \mathcal{L}$

$$
u_{l}(X)=\min \{E(l(U, X)), U \text { uniform }\}
$$

The superdifferentiability properties of such utilities have been studied in [8] where it is proved that

$$
\partial u_{l}(X)=\overline{\operatorname{co}}\left\{\partial_{x} l(U, X), U \in \mathcal{V}_{X}\right\}
$$

where $\overline{\mathrm{co}}$ denotes closed convex hull operation for the $L^{1}(\Omega, \mathcal{F}, \mathbb{P})$ topology and $\mathcal{V}_{X}$ denotes the set of uniformly distributed random variables such that $u_{l}(X)=\mathbb{E}(l(U, X))$. Note that since $\partial u_{l}(X)$ is a bounded subset of $L^{\infty}$, it is 
$\sigma\left(L^{1}, L^{\infty}\right)$ - compact. The class $\mathcal{U}$ therefore satisfies (H1) and (H2). If we further assume that for every compact $K$ of $\mathbb{R}$, one has

$$
\inf _{(t, x) \in[0,1] \times K, l \in \mathcal{L}} \partial_{x} l(t, x)>0
$$

then taking again $\Phi=1$, we claim that (H3) is also satisfied. Indeed, let $X \in E$ and consider the set

$$
V(X):=\left\{\frac{Z}{\mathbb{E}(Z)}, Z \in \bigcup_{l \in \mathcal{L}} \partial u_{l}(X)\right\} .
$$

This set is bounded in $L^{\infty}$ and thus $\sigma\left(L^{1}, L^{\infty}\right)$ relatively compact in $L^{1}$. To show that $V(X)$ is $\sigma\left(L^{1}, L^{\infty}\right)$ closed, since it is convex, it suffices to prove that it is strongly closed. Let us then suppose that $Y_{n}=Z_{n} / \mathbb{E}\left(Z_{n}\right)$ with $Z_{n} \in \partial u_{l_{n}}(X)$ for some $l_{n} \in \mathcal{L}$ converges in $L^{1}$ to some $Y$. Since $\mathbb{E}\left(Z_{n}\right)$ is bounded and bounded away from 0 , up to a subsequence, we may assume that $Z_{n}$ converges in $L^{1}$ to some $Z$ and that $l_{n}$ converges to $l$. Passing to the limit in the inequality

$$
u_{l_{n}}(Y)-u_{l_{n}}(X) \leq Z_{n} \cdot(Y-X), \text { for all } Y \in E
$$

and using the continuity of the evaluation maps, we obtain that $Z \in \partial u_{l}(X)$. Hence $Y=Z / \mathbb{E}(Z) \in V(X)$ which proves (H3).

\section{$3 \quad$ No-trade principle and efficient allocations}

\subsection{No-trade principle for sets of linear utilities}

We first consider families of linear utilities on $E$. Agent $i$ 's set of utilities is parametrized by $K_{i}$, a convex and $\sigma(F, E)$ compact subset of $F$. It is further assumed that the family $\left(K_{i}\right)_{i \in I}$ lies in a common hyperplane of $F$ : there exists $\Phi \in E$ such that $a_{i} \cdot \Phi=1$ for all $a_{i} \in K_{i}$ and all $i$. We show that no subset of agents can make a profitable trade if and only if $\bigcap_{i \in I} K_{i} \neq \emptyset$. The main part of the proof of the theorem that follows (that is the equivalence between 1 and 2), is an infinite dimensional version of Samet's results [30].

Theorem 3.1. The following assertions are equivalent:

1. There exists no $\left(X_{i}\right)_{i \in I} \in E^{I}$ with $\sum_{i \in I} X_{i}=0$ such that $a_{i} \cdot X_{i}>0$ for all $a_{i} \in K_{i}$ and all $i \in I$,

2. $\bigcap_{i \in I} K_{i} \neq \emptyset$, 
3. There exists $P \in F$ such that for all $i \in I, a_{i} \cdot X_{i}>0$ for all $a_{i} \in K_{i}$ implies $P \cdot X_{i}>0$.

Proof. - 1. implies 2. Assume 1. and that $\bigcap_{i \in I} K_{i}=\emptyset$. Let $K=$ $K_{1} \times K_{2} \ldots \times K_{I}$ and $L=\{(P, P, \ldots, P), P \in F\}$ be the diagonal of $F^{I}$. We then have $K \cap L=\emptyset$. Since $K$ is $\sigma\left(F^{I}, E^{I}\right)$ compact and convex and $L$ is $\sigma\left(F^{I}, E^{I}\right)$-closed convex, from Hahn-Banach's theorem, there exists $\left(X_{i}\right)_{i \in I} \in E^{I}$ and $c \in \mathbb{R}$ such that

$$
\sum_{i} a_{i} \cdot X_{i}>c \geq P \cdot\left(\sum_{i} X_{i}\right), \text { for all } P \in F, a_{i} \in K_{i}, i \in I
$$

From the right-hand side, we obtain that $\sum_{i \in I} X_{i}=0$ and $c \geq 0$. Let $\bar{a}_{i} \in \operatorname{argmin}_{a_{i} \in K_{i}} a_{i} \cdot X_{i}$ and $\bar{c}_{i}=\bar{a}_{i} \cdot X_{i}$. We claim that there exists $c_{i}<\bar{c}_{i}$ for all $i \in I$ with $\sum_{i} c_{i}=0$. If not $\sum_{i} c_{i}$ would have a constant sign (strictly negative) on $\left\{\left(c_{i}\right) \in \mathbb{R}^{I} \mid \bar{c}_{i}-c_{i}>0, \forall i \in I\right\}$ contradicting $\sum_{i \in I} \bar{c}_{i}>0$. Let $\tilde{X}_{i}=X_{i}-c_{i} \Phi$. We have $\sum_{i} \tilde{X}_{i}=0$ and $a_{i} \cdot \tilde{X}_{i}=a_{i} \cdot X_{i}-c_{i} a_{i} \cdot \Phi=a_{i} \cdot X_{i}-c_{i}>0$ for every $a_{i} \in K_{i}, i \in I$ contradicting 1 . Therefore $\bigcap_{i=I} K_{i} \neq \emptyset$.

- 2 implies 3. Indeed, any $P \in \bigcap_{i \in I} K_{i}$ fulfills 3 .

- 3. implies 1. If $\sum_{i \in I} X_{i}=0$ and $a_{i} \cdot X_{i}>0$ for all $a_{i} \in K_{i}$ and all $i \in I$, then $P \cdot X_{i}>0$ for all $i \in I$ contradicting $\sum_{i \in I} X_{i}=0$.

The hypothesis that there exists $\Phi \in E$ such that $a_{i} \cdot \Phi=1$ for all $a_{i} \in K_{i}$ and all $i$ is in particular verified for $\Phi=1$ when $\left(K_{i}\right)_{i \in I}$ are subsets of probabilities or densities interpreted as sets of possible priors of the agents. The equivalence between assertions 1 and 2 of theorem 3.1 provides an exante no-trade principle. By applying theorem 3.1 to various pairs $(E, F)$, one recovers most of the no-trade results in the literature. Samet [30] considers the case of $E=\mathbb{R}^{m}, F=E^{\prime}$ and of a collection of non-empty convex closed subsets of probabilities $\left(K_{i}\right)_{i \in I}$. Man-Chung $\mathrm{Ng}[22]$ considers $E=C(A)$ the set of continuous functions on a compact set $A$ endowed with the supnorm and $F=E^{\prime}$ the set of finite Borel measures on $A$ and a collection $\left(K_{i}\right)_{i \in I} \subset F$ of non-empty convex weak star compact subsets of probability measures. For a given probability space $(\Omega, \mathcal{F}, P)$, Dana and Riedel [12] consider $E=L^{\infty}(\Omega, \mathbb{R}), \quad F=L^{1}(\Omega, \mathbb{R})$ and $\left(K_{i}\right)_{i \in I}$ a collection of nonempty convex $\sigma\left(L^{\infty}, L^{1}\right)$ compact subsets of densities. Finally Billot et al [5] consider $E=B(\Omega, \mathcal{F})$ the space of real-valued bounded measurable functions on $\Omega, F=E^{\prime}=$ ba $(\Omega, \mathcal{F})$ the space of finitely additive measures on $(\Omega, \mathcal{F})$ 
and $\left(K_{i}\right)_{i \in I}$ a collection of non-empty weak star $(\sigma(F, E))$ closed, convex subsets of countably additive probability measures on $(\Omega, \mathcal{F})$.

\subsection{No-trade principle for sets of concave utilities and efficiency}

We next consider the case where agents' preferences are given by families of concave utilities $\mathcal{U}_{i}$. We recall that $X_{i} \in E$ is strictly preferred to $Y_{i} \in E$ by agent $i$, which will be denoted $X_{i} \succ_{\mathcal{U}_{i}} Y_{i}$, if $u_{i}\left(X_{i}\right)>u_{i}\left(Y_{i}\right)$ for every $u_{i} \in \mathcal{U}_{i}$. The next lemma characterizes the directions in which preferences are increasing in a neighborhood of $X_{i}$ and shows that locally these directions coincide with the directions in which the linear utilities associated to the family of compact sets $\left(V_{i}\left(X_{i}\right)\right)_{i \in I}$ are increasing.

Lemma 3.2. Let $X_{i} \in E$ and $Y_{i} \in E$ be given. The following are equivalent:

1. There exists $t_{0}>0$ such that for all $t \in\left(0, t_{0}\right], X_{i}+t Y_{i} \succ_{\mathcal{U}_{i}} X_{i}$,

2. there exists $t_{0}>0$ such that $X_{i}+t_{0} Y_{i} \succ_{\mathcal{U}_{i}} X_{i}$,

3. for any $P \in V_{i}\left(X_{i}\right), P \cdot Y_{i}>0$ (or equivalently $P \cdot Y_{i}>0$ for all $P \in \partial u_{i}\left(X_{i}\right)$ and $\left.u_{i} \in \mathcal{U}_{i}\right)$.

Proof. Let us first remark that since $u_{i}$ is concave, the map $t \rightarrow t^{-1}\left(u_{i}\left(X_{i}+\right.\right.$ $\left.\left.t Y_{i}\right)-u_{i}\left(X_{i}\right)\right)$ is nonincreasing on $(0, \infty)$. Hence $u_{i}\left(X_{i}+t_{0} Y_{i}\right)-u_{i}\left(X_{i}\right)>0$ implies that $u_{i}\left(X_{i}+t Y_{i}\right)-u_{i}\left(X_{i}\right)>0$ for all $t \leq t_{0}$. Therefore, assertions 1 and 2 are equivalent. Let us prove that 2 and 3 are equivalent. Let $Y_{i}$ fulfill 3 and $u_{i} \in \mathcal{U}_{i}$ be fixed. From $(\mathbf{H 1}) \partial u_{i}\left(X_{i}\right)$ is $\sigma(F, E)$ compact. As $P \cdot Y_{i}>0$ for all $P \in \partial u_{i}\left(X_{i}\right)$, there exists $m>0$ such that $\min _{\left\{P \in \partial u_{i}\left(X_{i}\right)\right\}} P \cdot Y_{i} \geq m$. Since $u_{i}$ is concave, it is well-known (see e.g. [33], Theorem 2.4.9) that for every $\left(X_{i}, Y_{i}\right) \in E^{2}$,

$$
\lim _{t \rightarrow 0^{+}} t^{-1}\left[u_{i}\left(X_{i}+t Y_{i}\right)-u_{i}\left(X_{i}\right)\right]=\min _{\left\{P \in \partial u_{i}\left(X_{i}\right)\right\}} P \cdot Y_{i},
$$

hence for every $u_{i} \in \mathcal{U}_{i}$, there exists $t_{u_{i}}>0$ such that if $t \leq t_{u_{i}}, u_{i}(X+t Y)-$ $u_{i}(X)>0$. Let $t>0$ be given and let

$$
W_{i}(t)=\left\{u_{i} \in \mathcal{U}_{i} \mid u_{i}\left(X_{i}+t Y_{i}\right)-u_{i}\left(X_{i}\right)>0\right\} .
$$

From what preceeds, $\mathcal{U}_{i}=\cup_{t>0} W_{i}(t)$. From (H2), the evaluation maps being continuous, $W_{i}(t)$ is open. From $(\mathbf{H} 2), \mathcal{U}_{i}$ is compact, hence there exists a finite subcovering of $\mathcal{U}_{i}, W_{i}\left(t_{1}\right), \ldots, W_{i}\left(t_{k}\right)$. Let $t_{0}=\min _{j} t_{j}$. We then have that $X_{i}+t_{0} Y_{i} \succ_{\mathcal{U}_{i}} X_{i}$. 
Conversely, let $Y_{i}$ fulfill 2. Then that there exists $t_{0}>0$ such that $X_{i}+$ $t_{0} Y_{i} \succ_{\mathcal{U}_{i}} X_{i}$. For any $u_{i} \in \mathcal{U}_{i}$, we have

$$
0>u_{i}\left(X_{i}\right)-u_{i}\left(X_{i}+t_{0} Y_{i}\right) \geq-t_{0} P \cdot Y_{i} \text {, for any } Y_{i} \in \partial u_{i}\left(X_{i}\right)
$$

Hence $P \cdot Y_{i}>0$ for any $u_{i} \in \mathcal{U}_{i}$ and $P \in \partial u_{i}\left(X_{i}\right)$, equivalently $P \cdot Y_{i}>0$ for any $P \in V_{i}\left(X_{i}\right)$ proving that 2 implies 3 .

A non linear no-trade principle now follows:

Theorem 3.3. The following assertions are equivalent:

1. There exists no $\left(Y_{i}\right)_{i \in I} \in E^{I}$ with $\sum_{i \in I} Y_{i}=0$ such that $X_{i}+Y_{i} \succ_{\mathcal{U}_{i}} X_{i}$ for all $i \in I$,

2. $\bigcap_{i \in I} V_{i}\left(X_{i}\right) \neq \emptyset$,

3. there exists $P \in F$ such that for all $i \in I, X_{i}+t_{i} Y_{i} \succ_{\mathcal{U}_{i}} X_{i}$ for some $t_{i}>0$ implies $P \cdot Y_{i}>0$.

Proof. Let us show that 1 implies 2. From lemma 3.2, if assertion 1 holds true, there exists no $\left(Y_{i}\right)_{i \in I} \in E^{I}$ with $\sum_{i \in I} Y_{i}=0$ such that $P \cdot Y_{i}>0$ for all $P \in V_{i}\left(X_{i}\right)$ and all $i \in I$. From theorem 3.1 and (H3), we have $\bigcap_{i \in I} V_{i}\left(X_{i}\right) \neq \emptyset$.

2 implies 3. Assume 2, then from theorem 3.1, there exists $P \in F$ such that for all $i, Q \cdot Y_{i}>0$ for all $Q \in V_{i}\left(X_{i}\right)$ implies $P \cdot Y_{i}>0$. From lemma 3.2, there exists $P \in F$ such that for all $i X_{i}+t_{i} Y_{i} \succ_{\mathcal{U}_{i}} X_{i}$ for some $t_{i}>0$ implies $P \cdot Y_{i}>0$. Finally, the fact that 3 implies 1 is obvious.

Let us remark that the condition $\bigcap_{i \in I} V_{i}\left(X_{i}\right) \neq \emptyset$ in the previous theorem is similar to the condition that appears in proposition 7 of Rigotti, Shannon and Strzalecki [29]. However, the framework of proposition 7 in [29] is finitedimensional (the analysis is then extended to the duality between real-valued bounded functions and finitely additive measures but this is still a special case of our paper) and preferences are assumed to be complete.

Remark 3.4. From assertion 3, it can be seen that the assertions above are also equivalent to: "there exists no $J \subset I$ and $\left(Y_{i}\right)_{i \in J} \in E^{J}$ with $\sum_{i \in J} Y_{i}=0$ such that $X_{i}+Y_{i} \succ_{\mathcal{U}_{i}} X_{i}$ for all $i \in J$ ".

Setting $\mathcal{U}:=\prod_{i \in I} \mathcal{U}_{i}$, we may now define $\mathcal{U}$-efficient allocations.

Definition 3.5. Let $\left(X_{i}\right)_{i \in I} \in \mathcal{A}(X)$, then $\left(X_{i}\right)_{i \in I}$ is $\mathcal{U}$-efficient if there is no $\left(Y_{i}\right)_{i \in I} \in \mathcal{A}(X)$ such that $Y_{i} \succ_{\mathcal{U}_{i}} X_{i}$ for every $i \in I$. 
Let us first remark that if for each $i$, there exists $u_{i} \in \mathcal{U}_{i}$ such that $\left(X_{i}\right)_{i \in I}$ is efficient for the economy with complete preferences represented by the $\left(u_{i}\right)_{i \in I}$, then $\left(X_{i}\right)_{i \in I}$ is $\mathcal{U}$ - efficient. If not there would exist $\left(Y_{i}\right)_{i \in I} \in \mathcal{A}(X)$ such that, for all $i Y_{i} \succ_{\mathcal{U}_{i}} X_{i}$ and in particular $u_{i}\left(Y_{i}\right)>u_{i}\left(X_{i}\right)$ contradicting the efficiency of $\left(X_{i}\right)_{i \in I}$ for the $\left(u_{i}\right)_{i \in I}$.

We next characterize $\mathcal{U}$-efficiency and state our first main result that provides a positive answer to the first question adressed by the paper.

Theorem 3.6. The following assertions are equivalent:

1. The allocation $\left(X_{i}\right)_{i \in I} \in \mathcal{A}(X)$ is $\mathcal{U}$-efficient,

2. there exists no trade $\left(Y_{i}\right)_{i \in I} \in E^{I}$ with $\sum_{i \in I} Y_{i}=0$ such that $X_{i}+Y_{i} \succ_{\mathcal{U}_{i}}$ $X_{i}$ for all $i \in I$,

3. $\bigcap_{i \in I} V_{i}\left(X_{i}\right) \neq \emptyset$,

4. there exists $\left(u_{i}\right)_{i \in I} \in \mathcal{U}$ for each $i$ such that $\left(X_{i}\right)_{i \in I}$ is efficient for the economy with complete preferences represented by the $\left(u_{i}\right)_{i \in I}$.

Proof. The fact that 1 . implies 2. follows directly from the definition of efficiency. The implication 2. $\Rightarrow 3$. follows from Theorem 3.3. Assertion 3. implies assertion 4. since $\bigcap_{i \in I} V_{i}\left(X_{i}\right) \neq \emptyset$ implies that for each $i$, there exists $u_{i} \in \mathcal{U}_{i}$ and $\lambda_{i}>0$ such that $\bigcap_{i \in I} \lambda_{i} \partial u_{i}\left(X_{i}\right) \neq \emptyset$ which implies 4 . The implication $4 . \Rightarrow 1$. is straightforward and was already discussed.

Corollary 3.7. Let utilities be linear and fulfill the hypotheses of subsection 3.1. Then the following assertions are equivalent:

1. Any allocation $\left(X_{i}\right)_{i \in I} \in \mathcal{A}(X)$ is $\mathcal{U}$-efficient,

2. there exists no $\left(X_{i}\right)_{i \in I} \in E^{I}$ with $\sum_{i \in I} X_{i}=0$ such that $a_{i} \cdot X_{i}>0$ for all $a_{i} \in K_{i}$ and all $i \in I$,

3. $\bigcap_{i \in I} K_{i} \neq \emptyset$.

Let agents have endowments $W_{i} \in E, i \in I$ with $X=\sum_{i} W_{i}$. When is no-trade efficient, in other words when is $\left(W_{1}, \ldots, W_{I}\right)$ efficient? The following corollary follows directly from theorem 3.6.

Corollary 3.8. The following assertions are equivalent:

1. No-trade is $\mathcal{U}$-efficient, 
2. $\bigcap_{i \in I} V_{i}\left(W_{i}\right) \neq \emptyset$

3. there exists $\left(u_{i}\right)_{i \in I} \in \mathcal{U}$ such that no-trade is efficient for the economy with complete preferences represented by the $\left(u_{i}\right)_{i \in I}$.

Remark 3.9. A further assumption that can be made is that $E$ is ordered by a closed convex cone $E_{+}$in $E$ with non nonempty interior, polar to a convex cone $F_{+}$in $F$ (the cone of nonnegative prices). Adding nonnegativity constraints in the model, agents choose consumptions in $E_{+}$and their utility functions defined on $E_{+}$, are assumed to be concave, super differentiable on the interior of $E_{+}$and monotone with respect to the order associated to $E_{+}$. Let $\mathcal{A}_{+}(X):=\left\{\left(X_{i}\right)_{i} \in I \in \mathcal{A}(X) X_{i} \in E_{+}, \forall i \in I\right\}$. Assuming (H2) and $\partial u_{i}(X) \in F$ and $\sigma(F, E)$ compact and $V_{i}(X) \sigma(F, E)$ compact at any interior point $X$ of $E_{+}$and any $i$, theorem 3.6 can be stated as: an interior allocation $\left(X_{i}\right)_{i \in I}$ is $\mathcal{U}$-efficient if and only if there exists $\left(u_{i}\right)_{i \in I}, u_{i} \in \mathcal{U}_{i}$ for each $i$ such that $\left(X_{i}\right)_{i \in I}$ is efficient for the economy with complete preferences represented by the $\left(u_{i}\right)_{i \in I}$.

\section{Equilibria and welfare theorems}

A price $P \in F$ supports the preferred set to $X_{i}$ if $Y_{i} \succ_{\mathcal{U}_{i}} X_{i}$ implies $P \cdot Y_{i}>$ $P \cdot X_{i}$. Supporting prices to a preferred set have appeared in the previous section in theorem 3.1 and lemma 3.2. The next lemma characterizes them.

Lemma 4.1. For $P \in F$, the following are equivalent

1. $Y_{i} \succ_{\mathcal{U}_{i}} X_{i}$ implies $P \cdot Y_{i}>P \cdot X_{i}$,

2. $\lambda P \in V_{i}\left(X_{i}\right)$ for some $\lambda>0$,

3. there exists $u_{i} \in \mathcal{U}_{i}$ such that $X_{i}$ maximizes $u_{i}(Y)$ s.t. $P \cdot Y \leq P \cdot X_{i}$.

Proof. To show that 1 implies 2, let $P \in F$ be such that $Y_{i} \succ_{\mathcal{U}_{i}} X_{i}$ implies $P$. $Y_{i}>P \cdot X_{i}$. Assume that $\lambda P \notin V_{i}\left(X_{i}\right)$ for all $\lambda>0$. Since from (H3), $V_{i}\left(X_{i}\right)$ is $\sigma(F, E)$ compact, from Hahn-Banach's theorem there exists $Z_{i} \in E$ such that

$$
\lambda P \cdot Z_{i} \leq 0<\min _{H \in V_{i}\left(X_{i}\right)} H \cdot Z_{i}
$$

Since $H \cdot Z_{i}>0$, for all $H \in V_{i}\left(X_{i}\right)$, from lemma 3.2, for $t>0$ sufficiently small $X_{i}+t Z_{i} \succ_{\mathcal{U}_{i}} X_{i}$ while $P \cdot Z_{i}<0$ contradicting assertion 1 .

2 implies 3 , since if $\lambda P \in V_{i}\left(X_{i}\right)$ for some $\lambda>0$, there exists $u_{i} \in \mathcal{U}_{i}$ such 
that $\lambda P \in \partial u_{i}\left(X_{i}\right)$. Hence $X_{i}$ maximizes $u_{i}(Y)$ subject to $P \cdot Y \leq P \cdot X_{i}$. Finally to show that 3 implies 1 , if 3 holds true, then there exists $u_{i} \in \mathcal{U}_{i}$ and $\lambda>0$ such that $\lambda P \in \partial u_{i}\left(X_{i}\right)$. Let $Y \succ_{\mathcal{U}_{i}} X_{i}$, we then have

$$
0>u_{i}\left(X_{i}\right)-u_{i}(Y) \geq P \cdot\left(X_{i}-Y\right)
$$

and therefore $P \cdot Y>P \cdot X_{i}$ proving 1 .

The second main result of the paper, the characterization of equilibria for the incomplete preferences follows directly from the previous lemma as well as the characterisation of the demand correspondence. We now turn to the definition of concepts of equilibria and their characterization.

Definition 4.2. An allocation $\mathcal{X}^{*}=\left(X_{i}^{*}\right)_{i \in I} \in \mathcal{A}(X)$ with a price $P^{*} \in F$, is a $\mathcal{U}$-equilibrium with transfer payments if for every $i, X_{i} \succ_{\mathcal{U}_{i}} X_{i}^{*}$ implies $P^{*} \cdot X_{i}>P^{*} \cdot X_{i}^{*}$. An allocation $\left(X_{i}^{*}\right)_{i \in I} \in \mathcal{A}(X)$ with a price $P^{*} \in F$, is a $\mathcal{U}$-equilibrium if for every $i, P^{*} \cdot X_{i}^{*}=P^{*} \cdot W_{i}$ and for every $i, X_{i} \succ_{\mathcal{U}_{i}} X_{i}^{*}$ implies $P^{*} \cdot X_{i}>P^{*} \cdot W_{i}$.

Theorem 4.3. The following are equivalent

1. $\left(\mathcal{X}^{*}, P^{*}\right)$ is a $\mathcal{U}$-equilibrium with transfer payments,

2. $\lambda P^{*} \in \bigcap_{i \in I}^{d} V_{i}\left(X_{i}^{*}\right)$ for some $\lambda>0$,

3. there exists $\left(u_{i}\right) \in \mathcal{U}$ such that $\left(\mathcal{X}^{*}, P^{*}\right)$ is an equilibrium with transfer payment of the economy with utilities $\left(u_{i}\right)$.

The following are equivalent:

1. $\left(\mathcal{X}^{*}, P^{*}\right)$ is a $\mathcal{U}$-equilibrium,

2. $\lambda P^{*} \in \bigcap_{i \in I}^{d} V_{i}\left(X_{i}^{*}\right)$ for some $\lambda>0$ and for every $i, P^{*} \cdot X_{i}^{*}=P^{*} \cdot W_{i}$,

3. there exists $\left(u_{i}\right) \in \mathcal{U}$ such that $\left(\mathcal{X}^{*}, P^{*}\right)$ is an equilibrium of the economy with utilities $\left(u_{i}\right)$.

The proof of Theorem 4.3 follows directly from lemma 4.1.

Remark 4.4. As in remark 3.9, the case of consumptions in $E_{+}$may be considered. Under the same assumptions as those of remark 3.9), one can state the second part of theorem 4.3 as interior $\mathcal{U}$-equilibria coincide with interior equilibria of the economies with utilities $\left(u_{i}\right)$ for some $\left(u_{i}\right) \in \mathcal{U}$.

We may now prove the welfare theorems for incomplete preferences. 
Theorem 4.5. The following assertions hold:

1. Any $\mathcal{U}$-equilibrium is $\mathcal{U}$-efficient.

2. Any $\mathcal{U}$-efficient allocation is a $\mathcal{U}$-equilibrium with transfer payments for some $P \in F$.

Proof. Proof of assertion 1. From theorem 4.3, any $\mathcal{U}$-equilibrium $\left(\mathcal{X}^{*}, P^{*}\right)$ is an equilibrium for the some economy with complete preferences $u_{i}, i \in I$, hence is efficient in that economy. From theorem 3.6, $\left(X_{i}^{*}\right)_{i \in I}$ is $\mathcal{U}$-efficient. Let us now prove assertion 2: if $\left(X_{i}^{*}\right)_{i \in I}$ is $\mathcal{U}$-efficient, from theorem 3.6, it is efficient for the some economy with complete preferences $u_{i}, i \in I$, hence there exists $P^{*}$, such that $\left(\left(X_{i}^{*}\right)_{i \in I}, P^{*}\right)$ is an equilibrium with transfer payments $u_{i}, i \in I$. From 4.3, $\left(\left(X_{i}^{*}\right)_{i \in I}, P^{*}\right)$ is a $\mathcal{U}$-equilibrium with transfer payments.

\section{Discussions of concepts}

\subsection{Other strict preferences}

Given the family of utilities $\mathcal{U}_{i}$, we have considered the strict incomplete preference defined by: $Y_{i} \succ_{\mathcal{U}_{i}} X_{i}$ if and only if $u_{i}\left(Y_{i}\right)>u_{i}\left(X_{i}\right)$ for all $u_{i} \in \mathcal{U}_{i}$.

Given $\mathcal{U}_{i}$, another preference may be considered : $Y_{i} \succeq_{\widetilde{\mathcal{U}}_{i}} X_{i}$ if and only if $u_{i}\left(Y_{i}\right) \geq u_{i}\left(X_{i}\right)$ for all $u_{i} \in \mathcal{U}_{i}$. The strict associated preference is $Y_{i} \succ_{\tilde{\mathcal{U}}_{i}} X_{i}$ if and only if $u_{i}\left(Y_{i}\right) \geq u_{i}\left(X_{i}\right)$ for all $u_{i} \in \mathcal{U}_{i}$ with a strict inequality for some $u_{i} \in \mathcal{U}_{i}$. Clearly $Y_{i} \succ_{\mathcal{U}_{i}} X_{i}$ implies that $Y_{i} \succ_{\tilde{\mathcal{U}}_{i}} X_{i}$.

Two concepts of efficiency may be associated to the order $Y_{i} \succeq_{\tilde{\mathcal{U}}_{i}} X_{i}$ :

1. $\left(X_{i}\right)_{i \in I}$ is strongly- $\widetilde{\mathcal{U}}$-efficient if there does not exist $\left(Y_{i}\right)_{i \in I} \in \mathcal{A}(X)$ such that $Y_{i} \succeq_{\tilde{\mathcal{U}}_{i}} X_{i}$ for all $i$, strictly for some $i$,

2. $\left(X_{i}\right)_{i \in I}$ is weakly- $\widetilde{\mathcal{U}}$ efficient if there does not exist $\left(Y_{i}\right)_{i \in I} \in \mathcal{A}(X)$ such that $Y_{i} \succ_{\tilde{U}_{i}} X_{i}$ for all $i \in I$.

Let us now discuss the relations between the different concepts. By definition, strong- $\widetilde{\mathcal{U}}$-efficiency implies weak- $\widetilde{\mathcal{U}}$-efficiency which implies $\mathcal{U}$ efficiency.

Proposition 5.1. Assume (H1-H3) If the $u_{i}$ are strictly concave, $\mathcal{U}$ effi-

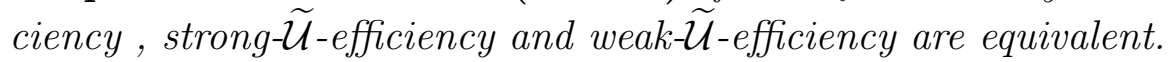


Proof. It remains to show that $\mathcal{U}$-efficiency implies strong- $\widetilde{\mathcal{U}}$-efficiency. Let $\left(X_{i}\right)_{i \in I}$ be $\mathcal{U}$-efficient and assume that there exists $\left(Y_{i}\right)_{i \in I} \in \mathcal{A}(X)$ such that $u_{i}\left(Y_{i}\right) \geq u_{i}\left(X_{i}\right)$ for all $i$ with a strict inequality for some $i$ and some $u_{i}$. Let $\left(Z_{i}=\left(Y_{i}+X_{i}\right) / 2\right) \in \mathcal{A}(X), u_{i}\left(Z_{i}\right) \geq u_{i}\left(X_{i}\right)$ for all $i$, and for all $i$ such that $Z_{i} \neq X_{i}, u_{i}\left(Z_{i}\right)>u_{i}\left(X_{i}\right)$ for all $u_{i} \in \mathcal{U}_{i}$. From remark 3.4, this contradicts $\mathcal{U}_{i}$-efficiency.

A counterexample Let us now give a counterexample where the set of strong- $\widetilde{\mathcal{U}}$-efficient allocations is strictly smaller than the set of $\mathcal{U}$ efficient allocations. Assume that there are $k$ states of the world with probabilities $\pi_{i}, i=1, \ldots, k$ and one good in each state of the world. A contingent claim $X=\left(x_{1}, \ldots, x_{k}\right)$ where $x_{j}$ is the amount to be received in state $j$ is identified to an element of $\mathbb{R}^{k}$. Hence $E=F=\mathbb{R}^{k}$. Let $\left(\mathcal{P}_{i}\right)_{i \in I}$ be a family of compact convex subsets of the probability simplex and denote by ri $\mathcal{P}_{i}$ the relative interior of $\mathcal{P}_{i}$. Let

$$
\mathcal{U}_{i}=\left\{u_{i}: \mathbb{R}^{k} \rightarrow \mathbb{R} \text { s.t. } u_{i}(X)=E_{\pi}(X)=\sum \pi_{i} x_{i}, \pi \in \mathcal{P}_{i}, X \in \mathbb{R}^{k}\right\}
$$

Let $Y_{i} \succeq_{\widetilde{\mathcal{u}}_{i}} X_{i}$ if and only if $E_{\pi}\left(Y_{i}\right) \geq E_{\pi}\left(X_{i}\right)$ for all $\pi \in \mathcal{P}_{i}$ and $Y_{i} \succ_{\tilde{\mathcal{u}}_{i}} X_{i}$ if and only if $E_{\pi}\left(Y_{i}\right) \geq E_{\pi}\left(X_{i}\right)$ for all $\pi \in \mathcal{P}_{i}$ with a strict inequality for some $\pi \in \mathcal{P}_{i}$. Let $X \in \mathbb{R}^{k}$ be the aggregate endowment. When utilities are linear, either all feasible allocations are efficient or there is no efficient allocation. From Dana and Le Van [11], any feasible allocation is $\widetilde{\mathcal{U}}$-efficient if and only if $\cap_{i}$ ri $\mathcal{P}_{i} \neq \emptyset$, while from section 3 , any feasible allocation is $\mathcal{U}$-efficient if and only if $\cap_{i} \mathcal{P}_{i} \neq \emptyset$. It is easy to construct examples where $\cap_{i}$ ri $\mathcal{P}_{i}=\emptyset$ and $\cap_{i} \mathcal{P}_{i} \neq \emptyset$.

An allocation $\left(X_{i}^{*}\right)_{i \in I} \in \mathcal{A}(X)$ with a price $P^{*} \in F$, is a $\tilde{\mathcal{U}}$-equilibrium if for every $i, P^{*} \cdot X_{i}^{*}=P^{*} \cdot W_{i}$ and $X_{i} \succ_{\tilde{\mathcal{U}}_{i}} X_{i}^{*}$ implies $P^{*} \cdot X_{i}>P^{*} \cdot W_{i}$. Note that any $\tilde{\mathcal{U}}$-equilibrium is a $\mathcal{U}$-equilibrium since $X_{i} \succ_{\mathcal{U}_{i}} X_{i}^{*}$ implies $X_{i} \succ_{\tilde{\mathcal{U}}_{i}} X_{i}^{*}$.

Proposition 5.2. If the $u_{i}$ are strictly concave, an allocation $\left(X_{i}^{*}\right)_{i \in I} \in$ $\mathcal{A}(X)$ with a price $P^{*} \in F$, is a $\tilde{\mathcal{U}}$-equilibrium if an only if it is a $\mathcal{U}$-equilibrium

Proof. It remains to show that if $\left(\mathcal{X}^{*}, P^{*}\right)$ is a $\mathcal{U}$-equilibrium, then it is a $\tilde{\mathcal{U}}$ equilibrium. Let $\left(\mathcal{X}^{*}, P^{*}\right)$ be a $\mathcal{U}$-equilibrium and assume that there exists for some $i, X_{i}$ such that $u_{i}\left(X_{i}\right) \geq u_{i}\left(X_{i}^{*}\right)$ for all $i$ with a strict inequality for some $u_{i}$ and $P^{*} \cdot X_{i} \leq P^{*} \cdot X_{i}^{*}=P^{*} \cdot W_{i}$. Let $Z_{i}=\left(X_{i}+X_{i}^{*}\right) / 2$. Then $P^{*} \cdot Z_{i} \leq P^{*} \cdot W_{i}$ and $Z_{i} \succ_{\mathcal{U}_{i}} X_{i}^{*}$. Indeed, since $X_{i} \neq X_{i}^{*}, u_{i}\left(Z_{i}\right)=u_{i}\left(\left(X_{i}+X_{i}^{*}\right) / 2\right)>u_{i}\left(X_{i}^{*}\right)$ for all $u_{i}$, contradicting the assumption that $\left(\mathcal{X}^{*}, P^{*}\right)$ is a $\mathcal{U}$-equilibrium of the economy with utilities $\left(u_{i}\right)$. 


\subsection{The case of second order stochastic dominance}

The aim of this paragraph is to extend theorems 3.6 and 4.3 to the case of the concave order in one dimension, a case in which neither (H2) nor (H3) are fulfilled. Let $(\Omega, \mathcal{F}, \mathbb{P})$ be a non-atomic probability space and consider the set of bounded real-valued random variables, $L^{\infty}=L^{\infty}((\Omega, \mathcal{F}, \mathbb{P}), \mathbb{R})$. Let $\mathcal{U}$ be the set of strictly concave increasing utilities on $\mathbb{R}$. For $X$ and $Y$ in $L^{\infty}$, let us denote $X \succ Y$ if and only if $\mathbb{E}(v(X))>\mathbb{E}(v(Y))$, for all $v \in \mathcal{U}$. As is well-know, the corresponding large preference, $X \succeq Y$, is defined by $X \succeq Y$ if and only if $E(v(X)) \geq \mathbb{E}(v(Y))$, for all $v$ concave nondecreasing. Let $\Phi=1$, and $V(X)=\{Y / \mathbb{E}(Y): Y \in \partial u(X), u \in \mathcal{U}\}$ be the set of normalized supergradients at $X \in L^{\infty}$.

Proposition 5.3. Let $\left(X_{i}\right)_{i \in I} \in \mathcal{A}(X)$. Then the following statements are equivalent:

1. $\left(X_{i}\right)_{i \in I}$ is strongly- $\widetilde{\mathcal{U}}$ efficient,

2. $\left(X_{i}\right)_{i \in I}$ is $\mathcal{U}$-efficient,

3. $\left(X_{i}\right)_{i \in I}$ is a comonotone allocation of $X$,

4. there exist continuous and strictly concave increasing functions $\left(u_{1}, \ldots, u_{I}\right)$ such that $\left(X_{i}\right)_{i \in I}$ is efficient for the corresponding expected utility economy,

5. $\bigcap_{i \in I} V\left(X_{i}\right) \neq \emptyset$.

Proof. The equivalence between assertions 1 and 2 follow from Proposition 5.1, that between 1, 3 and 4 from Carlier Dana and Galichon [7]. The equivalence between 4 and 5 follows from standard first order conditions for strictly concave utilities.

Given individual endowments $\left(W_{i}\right)_{i \in I}$ and defining $\mathcal{U}$-equilibria as previously, under some technical assumptions on the aggregate risk $X=\sum_{i \in I} W_{i}$, we have the following characterization

Theorem 5.4. Assume that $F_{X}$, the cumulative distribution function of $X$ is increasing and continuous, then the following assertions are equivalent:

1. $\left(\mathcal{X}^{*}, P^{*}\right)$ is a $\mathcal{U}$-equilibrium,

2. there exists $\lambda>0$ such that $\lambda P^{*} \in \bigcap_{i \in I}^{d} V_{i}\left(X_{i}^{*}\right)$ and $\mathbb{E}\left(P^{*} X_{i}^{*}\right)=$ $\mathbb{E}\left(P^{*} W_{i}\right)$ for every $i \in I$, 
3. there exists $\left(u_{i}\right)_{i \in I} \in \mathcal{U}^{I}$ such that $\left(\mathcal{X}^{*}, P^{*}\right)$ is an equilibrium of the economy with utilities $\left(u_{i}\right)_{i \in I}$.

Proof. Clearly, assertions 2 and 3 are equivalent and assertion 3 implies assertion 1. Let us now assume that $\left(\mathcal{X}^{*}, P^{*}\right)=\left(\left(X_{i}^{*}\right)_{i}, P^{*}\right)$ is a $\mathcal{U}$-equilibrium, which means that $\left(X_{i}^{*}\right)_{i} \in \mathcal{A}(X)$ and for every $i$, one has

$$
Y \succ X_{i}^{*} \Rightarrow \mathbb{E}\left(P^{*} Y\right)>\mathbb{E}\left(P^{*} X_{i}^{*}\right)=\mathbb{E}\left(P^{*} W_{i}\right) .
$$

First, we claim that the $X_{i}^{*}$ 's are $P^{*}$-measurable, since if not taking $Y_{i}:=$ $\mathbb{E}\left(X_{i}^{*} \mid P^{*}\right) \succ X_{i}^{*}$ as $\mathbb{E}\left(P^{*} Y_{i}\right)=\mathbb{E}\left(P^{*} X_{i}^{*}\right)$, we would derive a contradiction to (5.1). Hence $X_{i}^{*}=f_{i}\left(P^{*}\right)$ and $X=f\left(P^{*}\right)$ with $f=\sum_{i \in I} f_{i}$. This implies that $P^{*}$ is non-atomic since otherwise $X$ would have an atom, contradicting the assumption that $F_{X}$ is continuous. Since $P^{*}$ is non-atomic, we may find nonincreasing functions $g_{i}$ such that $Y_{i}:=g_{i}\left(P^{*}\right)$ has the same law as $X_{i}^{*}=f_{i}\left(P^{*}\right)$. If $X_{i}^{*} \neq Y_{i}$ ae, then $\left(X_{i}^{*}+Y_{i}\right) / 2 \succ X_{i}^{*}$ implying from (5.1) that $\mathbb{E}\left(P^{*} Y_{i}\right)>\mathbb{E}\left(P^{*} X_{i}^{*}\right)$ which contradicts Hardy-Littlewood's inequality $\mathbb{E}\left(P^{*} Y_{i}\right) \leq \mathbb{E}\left(P^{*} X_{i}^{*}\right)$. Hence it must be the case that $X_{i}^{*}=g_{i}\left(P^{*}\right)$ a.s.: the $X_{i}^{*}$ 's are nonincreasing functions of $P^{*}$. We may therefore find concave functions $u_{i}$ such that $P^{*} \in \partial u_{i}\left(X_{i}^{*}\right)$ for every $i$; furthermore, since $P^{*} \geq 0$, $u_{i}$ can also be taken nondecreasing. It remains to prove that the $u_{i}$ 's are strictly concave on the closed convex hull of the range of $X_{i}^{*}$. To prove this fact, given $Y \in L^{\infty}$, let $F_{Y}^{-1}$ be the generalized inverse (or quantile function) of the cumulative distribution function $F_{Y}$. Since $X=\sum_{i \in I} g_{i}\left(P^{*}\right)=: g\left(P^{*}\right)$ with all the $g_{i}$ 's nonincreasing, for Lebesgue almost-every $t \in[0,1]$, we have

$$
F_{X}^{-1}(t)=g\left(F_{P^{*}}^{-1}(1-t)\right)=\sum_{i \in I} g_{i}\left(F_{P^{*}}^{-1}(1-t)\right)=\sum_{i \in I} F_{X_{i}^{*}}^{-1}(t)
$$

Since we have assumed that $F_{X}$ is increasing, $F_{X}^{-1}$ is continuous. It thus follows from that previous identity that $F_{X_{i}^{*}}^{-1}$ is continuous or, equivalently $F_{X_{i}}^{*}$ is increasing for every $i$. If $u_{i}$ was affine one some nondegenerate interval $[a, b] \subset\left[\operatorname{essinf} X_{i}^{*}, \operatorname{esssup} X_{i}^{*}\right]$, then $P^{*}$ would be constant on the set $\left(X_{i}^{*}\right)^{-1}((a, b))$. Since $F_{X_{i}^{*}}$ is increasing, this set would have positive probability and $P^{*}$ would have an atom and we already know that this cannot happen. All the $u_{i}$ 's can therefore be chosen strictly concave (and thus increasing) implying assertion 2. 


\subsection{Relation to the literature}

Our paper belongs to the literature that deals with the following abstract problem: given a space with a preorder defined by a family of utility functions, under which conditions is it true that an undominated element maximizes a utility function in the family that is strictly increasing in the preorder. The definition of undominated element and that of a utility function increasing in the preorder $(X \succ Y$ implies $u(X)>u(Y))$ depends on how the strict preference is defined in terms of the family of utility functions. Most authors consider the strict preference defined in paragraph 5.1.

To our knowledge, there are no general results on the abstract problem although, it has been addressed in specific cases. A seminal example is the article of Arrow, Barankin and Blackwell [2], which provides a negative answer for a subset of an Euclidean space endowed with the componentwise order. The articles of Peleg [25] and and Peleg and Yaari [26] also provide a negative answer for a subset of $L^{\infty}$ and second order stochastic dominance. These papers nontheless show that the undominated elements that maximize a utility function increasing in the preorder are dense in the set of undominated elements. Recently a positive answer has been provided by Evren [16] for convex subsets of probabilities on a compact metric space for a strict order defined by convex families of vNM utilities defined by norm-compact sets of continuous utility indices.

Versions of the abstract problem have been addressed in different fields where they have received a positive answer. A first field is that of social planning with incomplete preferences. Let $E$ be a set of social alternatives for a society with $d$ agents. Agent's $i$ incomplete preferences are represented by the family of utilities $\mathcal{U}_{i}, i=1, \ldots, d$. One defines a social preorder by : for $(X, Y) \in E^{2}, X \succeq Y$ ( $X$ is prefered to $Y$ by the society) if and only if $u_{i}(X) \geq$ $u_{i}(Y)$ for any $u_{i} \in \mathcal{U}_{i}$ and any $i$. The social incomplete preorder on $E$ is thus represented by the family of utilities $\cup_{i} \mathcal{U}_{i}$ which is not convex in general. Its convexification is the set of weighted utilities $\sum_{i} \beta_{i} u_{i}, \sum_{i} \beta_{i}=1, u_{i} \in \mathcal{U}_{i}, \forall i$. Let $F \subset E$. One can ask under which conditions an undominated element of $F$ maximizes a weighted sum of utilities with strictly positive weights. Carroll [9] considers the case of lotteries on a finite set and convex families $\mathcal{U}_{i}$ of vNM utilities which coincide with their relative interior. His strict order is that of paragraph 5.1. Evren [16] applies his general result to this specific setting. His model thus generalizes Carroll's model, but his concept of efficiency is weaker. One can note that both models use linear utilities.

Another example is that of our paper: an exchange economy with given aggregate endowments. Let $E$ be the consumption space of an economy with $d$ agents. Agent's $i$ preferences are defined by a family of utilities $\mathcal{U}_{i}, i=$ 
$1, \ldots, d$. Aggregate endowment $W \in E$ is fixed. A feasible allocation is an element $X \in E^{d}, X=\left(X_{1}, \ldots, X_{d}\right)$ such that $\sum_{i=1}^{d} X_{i}=W$. Preferences are defined on the set of allocations and given by the set of weighted sums of utilities with strictly positive weights, each agent's utility being picked up from her utility set. One can ask whether it is true and under which conditions that a PO maximizes a weighted sum of utilities, each agent's utility being picked up from her utility set. Our paper provides sufficient conditions to obtain a positive answer that generalize Rigotti and Shannon [28] and Dana and Riedel [12] who assume that utilities are strictly concave. In the univariate case, a positive answer for the concave order is obtained for finite states spaces by Dana [10] and for non atomic spaces by Carlier et al [7]. A density result is proven in the multivariate case in Carlier et al [7].

A last example, in the field of non cooperative games, is that of the characterization of Nash equilibria when agents have incomplete preferences. Bade [4] shows that if each player's strategy space is a convex subset of a finite dimensional space and preferences are represented by the convex hull of a finite number of utilities and if the strict order is that of paragraph 5.1, then the set of Nash equilibria in pure strategy for incomplete preferences is the union of the sets of Nash equilibria that results when each agent's utility is picked up from her utility set. Evren [16] defines a refinement of the concept of pure-strategy Nash equilibrium. He introduces the concept of randomization-proof equilibrium in which each agent's equilibrium strategy is undominated in her set of mixed strategies. Under the assumptions on utilities of his general theorem, he shows that the set of randomization-proof equilibria is the union of the set of pure strategy equilibria that results when each agent's utility is picked up from her utility set.

\section{References}

[1] C.D. Aliprantis, K. Border, Infinite dimensional analysis. A hitchhiker's guide, Third edition, Springer, Berlin, 2006.

[2] K.J. Arrow, E.W. Barankin and D. Blackwell, Admissible points of convex sets, in: H.W. Kuhn and A.W. Tucker (Eds.), Contributions to the Theory of Games, Volume II, Princeton, Princeton University Press, 87-9, 1953.

[3] R. Aumann, Utility theory without the completeness axiom, Econometrica 30 (1962) 445-462. 
[4] S. Bade, Nash equilibrium in games with incomplete preferences, Economic Theory 26 (2005) 309-332.

[5] A. Billot, A. Chateauneuf, I. Gilboa and J.M. Tallon, Sharing beliefs: between agreeing and disagreeing the infinite case, Econometrica 68-3 (2000) 685-694.

[6] T. Bewley, Knightian Decision Theory: Part I, Decis. in Economics and Finance, 25 (2002) 79-110.

[7] G. Carlier, R.A. Dana and A. Galichon, Pareto efficiency for the concave order and multivariate comonotonicity, J. Econ. Theory 147 (2012) 207-229.

[8] G. Carlier, Differentiability properties of Rank-Linear Utilities, Journal of Mathematical Economics, 44 (2008) 15-23.

[9] G. Carroll, An efficiency theorem for incompletely known preferences, J. Econ. Theory 145 (2010) 2463-2470.

[10] R.A. Dana, Market behavior when preferences are generated by second order stochastic dominance, Journal of Mathematical Economics 40 (2004) 619-639.

[11] R.A. Dana and C. Le Van, Overlapping sets of priors and the existence of efficient allocations and equilibria for risk measures, Mathematical Finance 20-3 (2010) 327-339.

[12] R.A. Dana and F. Riedel, Intertemporal Equilibria with Knightian Uncertainty, downloadable at http: //www . imw . uni-bielefeld.de/papers/files/imw-wp-440.pdf, to appear in J. Econ. Theory.

[13] J. Dubra, F. Maccheroni, E.A. Ok, Expected utility theory without the completeness axiom, J. Econ. Theory 115 (2004) 118-133.

[14] P. Dybvig, Distributional Analysis of Portfolio Choice, Journal of Business, 61 (1988) 369-393.

[15] D. Easley M. O'Hara, Liquidity and valuation in an uncertain world, Journal of Financial Economics 97 (2010) 1-12.

[16] O. Evren, Scalarization Methods and Expected Multi-Utility Representations, working paper, 2012, downloadable at https://files.nyu.edu/oe240/public/research.html 
[17] I. Ekeland, R. Temam, Convex Analysis and Variational Problems, Classics in Mathematics, Society for Industrial and Applied Mathematics, Philadelphia, 1999.

[18] D. Gale, A. Mas-Colell, An equilibrium existence theorem for a General Model without Ordered Preferences, Journal of Mathematical Economics 2 (1975) 9-15.

[19] E. Jouini, H. Kallal, Efficient Trading Strategies in the Presence of Market Frictions, Review of Financial Studies 14 (2000) 343-369.

[20] R. D. Luce, H. Raiffa, Games and Decisions, John Wiley, New York, 1957.

[21] F. Maccheroni, Yaari dual theory without the completeness axiom, Economic Theory 23 (2004) 701-714.

[22] M.C. Ng, On the duality between prior beliefs and trading demands, J. Econ. Theory 109 (2003) 39-51.

[23] A. Mas-Colell, A equilibrium existence theorem without complete or transitive preferences, Journal of Mathematical Economics 1 (1974) 237-246.

[24] E.A. Ok, Utility representation of an incomplete preference relation, J. Econ. Theory, 104 (2003) 429-449.

[25] B. Peleg, Efficient Random Variables, Journal of Mathematical Economics 2 (1975) 243-252.

[26] B. Peleg, M.E. Yaari, A Price Characterisation of Efficient Random Variables, Econometrica 43 (1975) 283-292.

[27] M. Rothschild, J.E. Stiglitz, Increasing Risk, I. A Definition, J. Econ. Theory 2 (1970) 225-243.

[28] L. Rigotti, C. Shannon, Uncertainty and Risk in Financial Markets, Econometrica, 73 (2005) 203-243.

[29] L. Rigotti, C. Shannon, T. Strzalecki, Subjective beliefs and ex ante trade, Econometrica 76 (2008) 1167-1190.

[30] D. Samet, Common priors and separation of convex sets, Games and Economic behavior 24 (1998) 172-174. 
[31] L. S. Shapley, Equilibrium Points in Games with Vector Payoffs, Naval Research Logistics Quarterly 6 (1959) 57-61.

[32] J. von Neumann, O. Morgenstern, Theory of Games and Economic Behavior, Princeton University Press, Princeton, 1944.

[33] C. Zalinescu, Convex Analysis in General Vector Spaces, World Sci. Publ, River Edge, 2002. 